\title{
Componentes Reprodutivos e Produtivos no Rebanho de Corte da Embrapa Cerrados
}

\section{Concepta McManus ${ }^{1}$, Moacir Gabriel Saueressig ${ }^{2}$, Rui Arruda Falcão ${ }^{3}$, Germana Serrano $^{3}$, Kenia Régia Anasenko Marcelino ${ }^{3}$, Giane Regina Paludo ${ }^{1}$}

\begin{abstract}
RESUMO - O objetivo deste trabalho foi analisar os diversos fatores que influenciam o intervalo de partos (IEP), a fertilidade real (FR), razão do peso do bezerro ao nascer (RPN) ou desmame (RPD) em relação ao peso da vaca ao parto, no rebanho de gado mestiço da Embrapa Cerrados. Foram analisados dados de 4469 parições, coletados no período entre 1976 e 1999, usando os procedimentos GLM, CORR e PRINCOMP do SAS. As características incluíram as relações peso ao nascimento do bezerro/peso da vaca no parto (RPN), peso ao desmame do bezerro/ peso da vaca no parto (RPD) e a fertilidade real (FR), bem como a condição corporal (CC) e os pesos dos animais. A CC da vaca no parto (CCVP) não afetou a mortalidade, o peso ao nascer dos bezerros, ou o IEP. A FR elevou-se com o aumento da CCVP, seguindo a tendência oposta que o IEP. Verificaram-se menor IEP e maior FR com maiores valores de CC da vaca ao desmame (CCVD). O grupo genético do pai não influenciou nenhuma das características analisadas, enquanto o grupo da mãe foi significativo para todas as características estudadas. Somente o ano do nascimento influenciou a mortalidade dos bezerros. O ambiente (mês, ano e número do parto da mãe) e a genética (sexo) influenciaram significativamente os pesos ao nascer e ao desmame. As correlações entre as características estudadas foram, em geral, baixas e, às vezes, negativas para RPN e RPD, enquanto com FR foram mais altas. Os dois primeiros componentes explicam $56 \%$ da variação total entre as características examinadas.
\end{abstract}

Palavras-chave: fertilidade real, intervalo de partos, parâmetros reprodutivos, componentes principais, correlações

\section{Reproductive and Production Components for the Beef Herd of Embrapa Cerrados}

ABSTRACT - The objective of this work was to analyze the various factors which influence calving interval (CI), true fertility (TF), ratio of birth (RBW) and weaning weight (RWW) of the calf to the calving weight of the cow, using the crossbred herd of EMBRAPA Cerrados as a model. The data, from 4469 calvings, collected between 1976 and 1999, was analyzed using the GLM, CORR and PRINCOMP procedures of SAS. The reproductive parameters investigated include ratio of calf birth weight/calving weight of cow (RBW), calf weaning weight / calving weight of cow (RWW) and TF as well as body condition scores (BC) and weights. The correlations between the traits were, generally, low, and sometimes negative with RBW and RWW while with TF they were higher. BC at calving did not affect mortality nor calf birth weight but did affect the following calving interval. The FR increased with increased calving BC as did calving interval. A lower calving interval and higher true fertility was observed with higher weaning BC score. The genetic group of the bull did not influence any of the traits analyzed, which group of the cow influenced birth weight. The environment (month, year and number of parturition) and genetics (sex) significantly influenced birth and weaning weights. The first two principal components explain $56 \%$ of the total variation between the traits examined.

Key Words: calving interval, true fertility, reproductive parameters, principal components, correlations

\section{Introdução}

O crescimento sistemático da população brasileira acarreta uma demanda crescente de proteína animal. Com isso, há necessidade de melhorar substancialmente a produtividade do rebanho nacional, principalmente no que diz respeito ao desempenho reprodutivo, que é de fundamental importância na taxa de desfrute e na determinação de maior ou menor disponibilidade de animais para seleção (Szechy, 1995).

A pecuária de corte brasileira possui uma predominância de zebuínos, representando cerca de $85 \%$ dos genes que compõem o rebanho brasileiro. Este predomínio do grau de sangue zebuíno possibilitou a ocupação de grandes áreas nos cerrados (Carvalho, 1998). Como conseqüência dos sistemas de criação estabelecidos (condições naturais), onde os animais recebem com a máxima intensidade os efeitos diretos e indiretos do clima, o rebanho brasileiro apresenta o que se chama de tolerância ao ambiente tropical, numa adaptação durante a qual se perdeu qualidade produtiva. Este aspecto pode ser comprovado ao se observar a baixa taxa de desfrute do rebanho brasileiro, que se deve aos baixos índices produtivos e reprodutivos de nossa pecuária. 
O uso de intervalo de partos (IEP) nos processos de seleção tem sido um excelente parâmetro (Alencar et al., 1984), entretanto, Campello et al. (1999) e Alencar et al. (1997) salientam que, devido ao constante aprimoramento que a pecuária vem atravessando, surgiu a necessidade de parâmetros que pudessem auxiliar o melhorista na seleção de animais superiores, sendo destacados a fertilidade real (FR), as relações peso ao nascimento do bezerro/peso da vaca no parto (RPN) e peso ao desmame do bezerro/peso da vaca no parto (RPD).

A FR é a forma de avaliação de matrizes que envolve eficiência reprodutiva e habilidade materna (Campello et al., 1999). A habilidade materna, fator de fundamental importância no que diz respeito à FR, é um conjunto de atributos que a vaca deve possuir para fornecer o melhor desenvolvimento possível à sua cria, incluindo imunidade passiva, atenção, proteção e capacidade genética de adaptação (Carvalho et al., 1998).

A utilização dos parâmetros RPN e RPD, segundo Alencar et al. (1997), é justificada pelo fato de que, apesar de os cruzamentos resultarem em animais mais pesados, as vacas mais pesadas são as que produzem mais leite e consomem mais alimentos e podem não ser as mais eficientes. $\mathrm{O}$ objetivo deste trabalho foi investigar a relação entre as características reprodutivas de bovinos de corte do sistema da EMBRAPA Cerrados.

\section{Material e Métodos}

Foram coletados dados de 4469 parições, entre os anos de 1976 e 1999, de um rebanho mestiço de gado de corte da EMBRAPA Cerrados, localizada em Planaltina (DF). O rebanho foi criado em sistema exclusivo de pastejo, utilizando-se pastagens nativas e cultivadas e suplementação com sal mineral. $\mathrm{O}$ sistema de reprodução foi o de monta natural, com estação de monta compreendida nos meses de novembro a fevereiro.

Foram analisadas as seguintes características: peso da vaca no parto (PVP), condição corporal (CC) da vaca no parto (CCVP), peso do bezerro ao nascer $(\mathrm{PBN})$, peso da vaca ao desmame (PVD), CC da vaca ao desmame(CCVD), peso do bezerro ao desmame (PBD), com informação sobre a data do parto, grupo genético da mãe e pai e sexo do bezerro. Por meio desses dados foi possível obter a fertilidade real (FR), a relação entre o peso ao nascer do bezerro e da vaca no nascimento (RPN) e a relação entre o peso do bezerro e o da vaca ao desmame (RPD). A FR foi estimada de acordo com a fórmula descrita por Lobo et al. (1994), citada e utilizada por Campello et al. (1999):

$$
\frac{\mathrm{FR}=\mathrm{PBD} \times 365 \text { dias; }}{\text { IEP }}
$$

em que: PBD = peso do bezerro ao desmame $(\mathrm{kg})$; IEP = intervalo de partos (dias); 365 dias = índice em base anual.

O RPN e o RPD foram calculados pelas fórmulas usadas por Alencar et al. (1997):

$$
\mathrm{RPN}=\frac{\mathrm{PBN}}{\mathrm{PVP}} \text { e RPD }=\frac{\mathrm{PBD}}{\mathrm{PVP}} \mathrm{RPD} 2=\frac{\mathrm{PBD}}{\mathrm{PVD}}
$$

em que: $\mathrm{PBN}=$ peso do bezerro ao nascer; $\mathrm{PBD}=$ peso do bezerro ao desmame; $\mathrm{PVP}=$ peso da vaca ao parto; $\mathrm{PVD}=$ peso da vaca ao desmame.

A CC da vaca foi medida numa escala de 1 a 10 , em que 1 corresponde a um animal muito magro e 10 , a um obeso.

Os dados foram analisados usando os procedimentos estatísticos do Statistical Analysis System (1999). As características foram testadas para normalidade (todas foram normalmente distribuídas) usando o procedimento UNIVARIATE antes de realizar a análise de variância. Os dados referentes a FR e RPD foram analisados usando o procedimento GLM, segundo o modelo matemático:

$$
\begin{gathered}
Y_{\mathrm{ijklmno}}=\mu+\mathrm{M}_{\mathrm{i}}+\mathrm{A}_{\mathrm{j}}+\mathrm{S}_{\mathrm{k}}+\mathrm{GG}_{1}+\mathrm{N}_{\mathrm{m}}+\mathrm{CCVP}_{\mathrm{n}} \\
+\mathrm{b}_{1}\left(\mathrm{PVP}_{\mathrm{ijklmn}}-\mathrm{PVP}\right)+\mathrm{b}_{2}\left(\mathrm{PBN}_{\mathrm{ijklmn}}-\mathrm{PBN}\right) \\
\mathrm{p}+\mathrm{e}_{\mathrm{ijklmno}}
\end{gathered}
$$

em que: $Y_{i j k l m n o p}=$ observação do indivíduo p nascido no mês(M) i, do ano (A) j, do sexo(S) k, do grupo genético $(\mathrm{GG}) 1$, do número do parto $(\mathrm{N}) \mathrm{m}$, da condição corporal da vaca no parto (CCVP) n, sendo $\mathrm{i}=$ janeiro a dezembro, $\mathrm{j}=1976,1977, \ldots, 1999, \mathrm{k}=$ 1(macho), 2 (fêmea), $1=$ Zebu, $3 / 4$ Zebu, $1 / 2$ Zebu, 3/4 Canchim, $1 / 2$ Canchim, 3/4 Holandês, $1 / 2$ Holandês, $3 / 4$ Simental, $1 / 2$ Simental, $1 / 2$ Pardo Suiss, 1/2 Mocho Nacional, e Mocho Nacional; $\mathrm{m}=1, \ldots, 12 ; \mathrm{n}=1, \ldots 10$; $\mathrm{PVP}_{\mathrm{ijklmn}}=$ peso da vaca no parto; $\mathrm{b}_{1}=$ coeficiente de regressão linear associado à covariável $\mathrm{PVP}_{\mathrm{ijklmn}}$; $\mathrm{PBN}_{\mathrm{ijklmn}}=$ peso do bezerro ao nascer, $\mathrm{b}_{2}=$ coeficiente de regressão linear associado à covariável $\mathrm{PBN}_{\mathrm{ijklmn}} ; \mathrm{e}_{\mathrm{ijklmn}}=$ erro aleatório associado a cada observação, suposto normalmente distribuído e independente, com média zero e variância $\sigma^{2}$. As regressões quadráticas para PBN e PVP foram investigadas mas não foram significativas e posteriormente tiradas da análise. 
As características de PBN, PBD, mortalidade, RPN e RPD2 foram analisadas com o mesmo modelo excluindo PBN como covariável. Com IEP, o sexo do bezerro e grupo genético (GG) do pai foram eliminados do modelo e com PVD o GG do Pai e PBN. Nos casos de CCVP e CCVD os fatores GG do pai do bezerro, PVP, CCVP e PBN foram eliminados e com PVP o sexo do bezerro também foi eliminado.

Foram feitos também estudos das correlações entre as características e dos componentes principais usando os procedimentos CORR e PRINCOMP do SAS (1999) respectivamente.

\section{Resultados e Discussão}

O resumo da análise de variância está apresentado na Tabela 1. Mês e ano do parto, grupo genético do bezerro, número do parto da vaca e peso da vaca no parto, em geral, afetaram as características estudadas. A CC da vaca não afetou as características estudadas e, separando o grupo genético do bezerro em grupo da mãe e do pai, a raça do pai não influenciou as características estudadas, enquanto o grupo da mãe (Tabela 2) foi significativo para todas as características, exceto RPD2. Alencar (1988) não notou diferenças devido ao grupo genético do pai entre os RPDs enquanto Euclides Filho et al. (1992) acharam diferenças devido ao grupo genético da vaca, quando acasalada com touros Nelore.

Os animais oriundos de partos de vacas zebuínas foram os mais leves ao nascer e ao desmame, enquanto os de partos de vacas mestiças com Bos Taurus foram mais pesados. As vacas mestiças tiveram a tendência de ser mais pesadas, embora as vacas Gir também fossem mais pesadas tanto no parto quanto ao desmame. A CC das vacas zebuínas (Nelore) foi significativamente mais baixa de que a dos outros grupos genéticos. O IEP das vacas mestiças teve a tendência de ser mais alto que o das vacas puras que, combinado com o peso do bezerro mais alto ao desmame, resultou em FRs e RPNs mais altos para este grupo. As vacas dos grupos $1 / 2$ Holandês $\mathrm{X} 1 / 2$ Zebu e Canchim tiveram valores mais altos para RPD.

O rebanho obteve média para o PBN de $25,51 \pm 5,51 \mathrm{~kg}$, valor abaixo dos encontrados por Széchy et al. (1995) em sua revisão sobre gado Nelore, onde cita valores entre 26,5 e $31,50 \mathrm{~kg}$ como esperados para a raça. Foi uma característica influenciada significativamente $(\mathrm{p}<0,01)$ pelo mês e ano de parto, sexo do bezerro, ordem de parto, grupo genético da mãe, não sendo influenciado pelo grupo genético do pai e pela CCVP. Alencar et al. (1997), trabalhando com bovinos da raça Nelore, obtiveram bezerros com $27,9 \pm 0,1 \mathrm{~kg}$ de peso ao nascimento, tendo sido a característica influenciada significativamente $(p<0,01)$ pelo ano do parto, mês do parto, ordem de parição e sexo do bezerro. Foi observada alta influência significativa $(\mathrm{p}<0,01)$ do mês e ordem de parto, mês de parto e sexo do bezerro, e grupo genético do bezerro, sobre o peso ao nascer, em toda a literatura consultada. Em trabalho desenvolvido por Széchy et al. (1995) não foi verificada diferença significativa entre os meses de parto para PBN, o que é justificado pela regularidade de alimentação dos animais ao longo dos meses do ano, além de uma adaptabilidade a curtas restrições alimentares. Isto também foi evidenciado para o rebanho da Embrapa Cerrados (Tabela 1), porém os animais sofreram restrição alimentar, durante o período da seca, maior que o de Széchy et al.(1995), resultando em bezerros mais pesados quando nascidos nos meses de maio $(29,34 \mathrm{~kg})$, período de elevada disponibilidade de forragem. A ordem de parto apresentou variação irregular ao longo das parições, sendo verificada tendência de queda do PBN a partir da oitava parição. Os bezerros machos $(27,07 \pm 5,52 \mathrm{~kg})$ foram mais pesados que as fêmeas $(26,17 \pm 5,95 \mathrm{~kg})$, o que está de acordo com a literatura consultada, justificado pela ação precoce da testosterona que determina uma taxa metabólica mais acentuada do feto durante o período de gestação (Széchy et al., 1995).

A não observação de influência do grupo genético do reprodutor sobre o PBN, pode ter sido ocasionada pela proximidade de parentesco entre os mesmos, já que esse fator é considerado como tendo influência por Campello et al. (1999). Em relação às matrizes foi verificada influência significativa $(p<0,01)$, o que demonstra a possibilidade de se alterar o valor de PBN pela seleção das reprodutoras.

O peso médio ao desmame observado no rebanho foi de $132,99 \pm 35,39 \mathrm{~kg}$, sendo bem abaixo dos $171 \mathrm{~kg}$, $178 \mathrm{~kg}$ e $185 \mathrm{~kg}$ encontrados em rebanho de Nelore por Alencar et al. (1984), Ayala et al. (1991) e Campello et al. (1999), respectivamente.

O PBD foi influenciado significativamente $(p<0,01)$ pelo mês do parto, ano, sexo do bezerro e ordem de parto. O resultado obtido está de acordo com a literatura consultada, onde é verificada a influência da disponibilidade de forragem ao longo do 


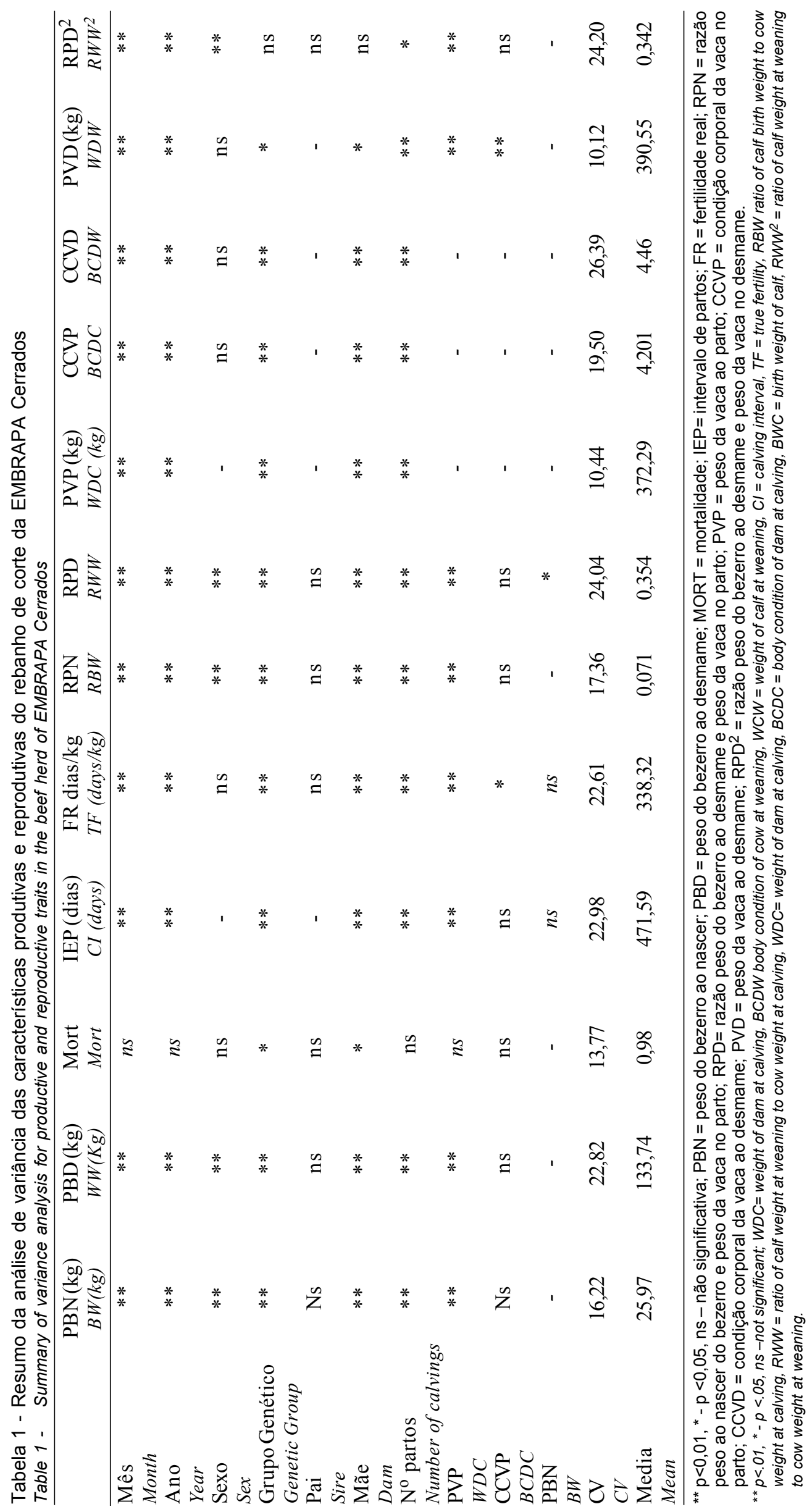




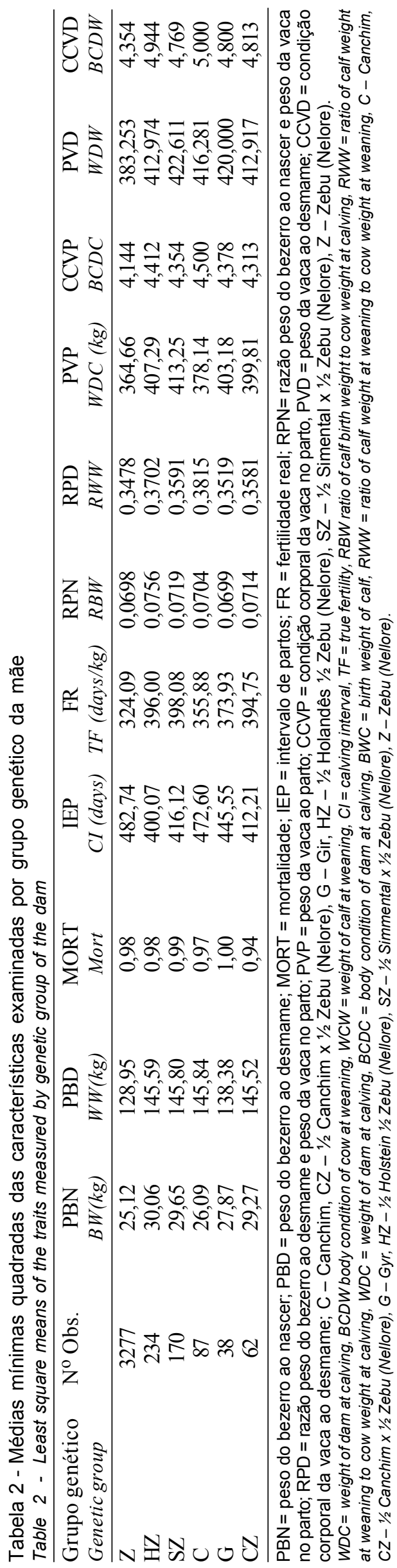

mês e ano em relação ao peso ao desmame, sendo a maior média encontrada para PBD no trabalho, relativa ao mês de maio $(150,89 \pm 43,52 \mathrm{~kg})$, coincidindo com o período de boa disponibilidade de forragem. A influência da ordem de parto foi irregular, não permitindo fazer uma avaliação da relação entre habilidade materna e peso ao desmame, o que contrasta com trabalho de Campello et al. (1999), no qual vacas de maior ordem de parto desmamam bezerros mais pesados devido ao aumento da habilidade materna. Os machos (135 kg) foram desmamados com um peso maior em relação às fêmeas $(132 \mathrm{~kg})$, o que vem confirmar o maior potencial de ganho de peso dos machos em relação às fêmeas, fato esse de consenso em toda a literatura consultada.

A não influência no PBD pelo efeito do reprodutor e da matriz, provavelmente deve-se ao nível de proximidade de parentesco entre os mesmos e à falta de alimento e manejo, que impediu a expressão de ganho de peso adequado dos animais superiores.

A mortalidade do rebanho foi de $2 \%$, sendo considerada baixa e influenciada significativamente apenas pelo ano do parto, o que caracteriza o rebanho como homogêneo em relação à rusticidade e adaptação ao local de criação. A influência do ano na mortalidade, tendo como idade de maior risco a de 22 meses, mostra uma falha de manejo alimentar ou sanitário do rebanho em alguns anos, devendo, portanto, ser reavaliado a fim de evitar queda nos índices de produtividade do rebanho. O rebanho é bastante longevo, com vacas apresentando até 14 parições.

A média geral do IEP foi de 15,7 meses $(471 \pm 140$ dias), sendo menor que a da maioria da literatura consultada para rebanho de corte (Martins \& Salomani, 1990, Pereira et al., 1991). Tal fato pode ser justificado pela razoável condição nutricional do rebanho, em que a média da CCVP foi de 4,15 $\pm 1,0$. Isso mostra que não houve restrição severa de alimentação durante o ano, o que possibilitou a vaca manter uma CC, no momento e após a parição, adequada para uma volta rápida à vida reprodutiva. Autores que obtiveram IEP similares ou menores que o deste trabalho justificaram como sendo devido à eficiente suplementação fornecida nos períodos de baixa qualidade das pastagens (Benevides et al., 1997; Campello et al., 1999). O elevado desviopadrão para o IEP $( \pm 140$ dias $)$ mostra uma possibilidade de se reduzir ainda mais esse parâmetro, melhorando o manejo do rebanho.

A literatura mostra que para se obterem melhores índices de natalidade no rebanho, diminuindo, portan- 
to o IEP, deve-se manter as matrizes com boa CC no momento do parto e após o mesmo (Fries \& Albuquerque, 1999), devendo-se evitar tanto animais em estado depauperado de alimentação, quanto animais obesos, que são tão ineficientes reprodutivamente quanto animais com restrição alimentar.

O IEP sofreu influência significativa $(\mathrm{p}<0,01)$ do mês de parto, ano, ordem de parto e raça da mãe $(\mathrm{p}<0,05)$. A influência do mês do parto está relacionada com a disponibilidade de forragem, responsável pela manutenção de bons níveis de nutrição e rápida volta à vida reprodutiva pós-parto. O maior IEP médio observado foi em março (493 dias) e o menor em outubro (421 dias). Também foi verificada uma diminuição do IEP com o aumento da ordem de parto até a $14^{\mathrm{a}}$ parição, provavelmente devido a seleção à qual os animais são submetidos, restando apenas aqueles superiores reprodutivamente. Esse resultado está de acordo com os obtidos por Széchy et al. (1995) e Campello et al. (1999). A influência da mãe no IEP se deve ao fato de que boas matrizes geralmente têm filhas que serão precoces e boas reprodutoras (Fries \& Albuquerque, 1999).

A FR (peso do bezerro à desmama por dias de intervalo de partos) foi influenciada significativamente pelo mês de parto, ano, grupo genético e ordem de parto. O mês de maior FR foi agosto $(130,1 \mathrm{~kg})$, sendo que em maio houve a maior média de peso ao desmame. Houve um alto IEP observado nesse mês, que resultou no FR mais baixo. O sexo da cria mostrou uma tendência de maiores FR para as vacas com bezerros machos, onde a FR foi de $113,9 \mathrm{~kg}$, contra $111,85 \mathrm{~kg}$ para vacas com bezerras. Quando o bezerro era macho houve uma tendência de ter um maior IEP, o que pode explicar porque a diferença entre FR entre vacas com bezerros machos e as com bezerras fêmeas não foi maior. Este maior IEP compensa o maior peso dos bezerros machos. $\mathrm{O}$ grupo genético tem influência, pois a habilidade materna é um atributo que se destaca de uma raça em relação a outra, possibilitando que alguns animais sejam mais aptos a criar seu bezerro de maneira a expressar todo o seu potencial produtivo (Campello et al. 1999). A influência da ordem de parto correspondeu à encontrada por Campello et al. (1999), observandose aumento da FR, de acordo com o aumento da ordem de parto.

A média dos valores encontrados para FR $(113,68$ $\pm 40,19 \mathrm{~kg}$ ) está bem abaixo dos valores citados na literatura. Em trabalho de Campello et al. (1999), foi encontrada uma média de $184,69 \mathrm{~kg}$, com erropadrão de $37,09 \mathrm{~kg}$, enquanto Gonçalves et al. (1996) encontraram uma média de $151,47 \mathrm{~kg}$, também acima do encontrado no presente trabalho. Esse baixo valor de FR não deveria ser observado, já que os valores de IEP foram baixos, podendo ser justificado pelo baixo peso ao desmame dos bezerros, provavelmente pela não seleção do rebanho com base nesse parâmetro de estimativa de eficiência durante os anos do experimento, o que não priorizou a obtenção de bezerros mais pesados ao desmame. Isto pode ser decorrente da época na qual os bezerros foram desmamados, do grupo genético do bezerro, ou simplesmente, devido a falhas no sistema de manejo (alimentação ou trato sanitário).

As médias dos valores do peso do bezerro ao nascer/peso da vaca no parto (RPN) $(0,070 \pm 0,013) \mathrm{e}$ do peso do bezerro ao desmama/peso da vaca no parto (RPD) $(0,354 \pm 0,094)$ foram bastante próximas dos valores encontrados por Alencar et al. (1997), os quais observaram em rebanho Nelore média de RPN de 0,0693 $\pm 0,0002$ e RPD de 0,427 $\pm 0,002$. O menor valor de RPD encontrado no trabalho permite concluir que o rebanho estudado foi menos eficiente, no que se refere à produção de quilos de bezerro desmamado por quilograma de vaca ao parto, que o rebanho analisado por Alencar et al. (1997); isto pode ser devido ao menor tamanho das matrizes (média de $369,94 \pm 60,16 \mathrm{~kg}$ ) em relação ao citado em seu trabalho $(406 \pm 1 \mathrm{~kg})$, já que os valores para peso ao nascer e peso a desmame foram inferiores.

O RPN sofreu influência significativa do mês, ano, mãe e ordem de parto. A maior média mensal do RPN foi obtida em novembro $(0,076)$, devido ao peso do bezerro, que nessa época é maior em relação aos demais períodos do ano. $\mathrm{O}$ ano tem efeito, pois as condições que influenciam o peso do bezerro e o da vaca oscilam de acordo com o ano. O efeito da mãe é pronunciado, pois características de peso na idade adulta e peso de bezerro são bastante influenciadas pelo genótipo passado pela mãe. Houve uma tendência de diminuir o RPN com o aumento da ordem de parição, provavelmente porque, mesmo havendo a geração de bezerros maiores pelas vacas de maior ordem (Alencar et al., 1997), há um aumento considerável do peso dessas matrizes ao longo dos anos. $\mathrm{O}$ efeito do sexo é claro ao se observar que é de consenso na literatura que bezerros machos tendem a nascer mais pesados, o que fez com que a média para as fêmeas fosse de 0,069 e para os machos de 
0,073. O RPD sofreu influência do mês, ano, sexo, ordem de parto, não tendo sido observada influência dos fatores genéticos. A influência do mês se deve ao fato de que o bezerro que tiver condições de pastejar por maior tempo em pasto de melhor qualidade nutricional e de maior disponibilidade, será desmamado com maior peso do que aquele desmamado nos períodos críticos de alimentação. (Alencar et al., 1997; Campello et al., 1999; Fries \& Albuquerque, 1999). O efeito do sexo é devido ao maior e mais rápido ganho de peso dos animais machos, de consenso na literatura, o que provoca uma RPD de 0,344 para fêmeas, contra 0,364 para machos. Foi observado que, quanto maior a ordem da vaca, menor o RPD, devido ao aumento do peso da vaca ser superior ao obtido pela maior habilidade materna, fato que se contrapõe ao trabalho de Alencar et al. (1997).

O processo de seleção deve objetivar a obtenção de um genótipo bovino adequado a um sistema de produção de ciclo curto, sem aumentar as exigências nutricionais, alterando as formas das curvas de crescimento corporal e de desenvolvimento/maturação sexual, mantendo ou reduzindo os tamanhos adultos, necessidade de mantença, a idade e peso de terminação ou acabamento de carcaças. Existem muitas características produtivas e reprodutivas que podem ser usadas pelos criadores para seleção dentro de seu rebanho.

Segundo Randel (1990), a CC é uma maneira eficiente de se avaliar o estado nutricional do bovino, pois influi diretamente nos valores produtivos e reprodutivos. Animais submetidos a baixos níveis nutricionais possuem menor peso ao desmame, retardam a idade à puberdade, possuem grande período de anestro pós-parto e período de serviço, resultando em maiores IEP e menor valor de FR.

As médias estimadas neste estudo para CCVP e CCVD foram de 4,16+1,04 e 4,41+1,30, com coeficientes de variação de 25,0 e $29,5 \%$, respectivamente. Estas médias foram consideradas satisfatórias, mesmo estando abaixo do ideal, de 5 a 7 . A CCVP e a CCVD foram afetadas significativamente $(p<0,01)$ pelos fatores ambientais (mês, ano e ordem de parições). A maior CCVP média $(5,30)$ observada é referente ao mês de junho, talvez devido à boa condição corporal resultante da época de chuvas anteriores, aliadas à suplementação alimentar fornecida aos animais nesse período. Vacas que pariram de janeiro a junho apresentaram a CCVP mais alta, enquanto as que pariram de maio a janeiro, apresentaram a CCVD mais elevada, provavelmente por receberem abundância e maior qualidade de pastagem no período. No decorrer dos anos ocorreu uma melhoria nos valores de CCVP e CCVD, fato verificado pela melhoria das condições de criação após o ano de 1990. A CCVP aumentou até a $3^{\mathrm{a}}$ parição e permaneceu no mesmo patamar até a $11^{\mathrm{a}}$ parição, enquanto a CCVD manteve-se estável da $1^{\mathrm{a}}$ a $11^{\mathrm{a}}$ parição. As vacas mais idosas tendem a ser mais pesadas (Alencar et al., 1997), o que pode influenciar este aumento até a $11^{\text {a }}$ parição. Semmelmann (2001), em pesquisa com lotes de novilhas Nelore, a CC de novilhas que emprenharam ou não aos 17-18 meses foi de 3,90 versus 3,81 no início do acasalamento, e 4,12 versus 4,01 para condição corporal no final do acasalamento. Gonzalez et al. (1993), obtiveram um período de serviço de 42,2 dias para novilhas mestiças com elevada condição corporal (>3) contra 81,0 dias para novilhas com baixa condição corporal, menor que $3(\mathrm{P}<0,05)$.

Houve influência significativa $(\mathrm{P}<0,01)$ do grupo genético do bezerro sobre a CCVP e a CCVD (Tabela 1). Não houve influência significativa do sexo da cria, grupo genético do pai e grupo genético da mãe sobre a CCVP e a CCVD. Resultados similares foram obtidos na literatura, onde a CCVP e a CCVD são relacionadas mais a fatores ambientais e de manejo do que a valores genéticos.

A CCVP não afetou a mortalidade e o peso ao nascer dos bezerros, embora exista a tendência de aumentar o peso ao nascer com aumento na CCVP. A CCVP afetou significativamente $(\mathrm{P}<0,05)$ o IEP seguinte. O IEP aumentou com a CCVP até CC 4 , ficou estável até CCVP 7 e depois diminuiu. Isto pode ser devido ao fato de vacas mestiças não necessitarem de grande quantidade de gordura para reproduzir bem ou muita gordura influencia negativamente a reprodução nessas vacas.

A FR elevou-se com o aumento da CCVP, seguindo a mesma tendência que o IEP. Foram verificados menor IEP e maior FR em maiores valores de CCVD. A CCVP não afetou a RPN e a RPD, embora exista tendência de ambos diminuírem com o aumento da CCVP. Com o incremento da CCVD, ocorreu o aumento da RPD.

Vacas com maior $\mathrm{CC}$ ao desmame foram mais pesadas e apresentaram maior PBD. Neste trabalho, verificou-se que, apesar de a CC ser considerada um indicador de eficiência do manejo nutricional e reprodutivo de rebanhos, não pode ser vista como 
principal fator determinante da eficiência reprodutiva, como cita boa parte da literatura, não excluindo sua influência bastante marcante em vacas primíparas. $\mathrm{O}$ resultado obtido se contrapõe à maioria dos autores consultados, que consideram a CC como sendo o fator mais limitante para o rápido aparecimento do cio, conseqüentemente menor período de serviço e menor IEP (Gonzalez et al., 1993).

Segundo Randel (1990), dietas com baixo nível energético, durante a fase final de gestação, levam a baixos índices reprodutivos, mesmo oferecendo dietas adequadas pós-parto. Pela literatura consultada parece claro que a ineficiência alimentar presente na maioria dos rebanhos brasileiros, que leva a baixas condições corporais, corresponde, se não ao principal, a um dos maiores problemas a serem resolvidos na pecuária brasileira, ficando difícil a proposição de seleção do rebanho para características de crescimento animal (ganho diário de peso, precocidade, conversão alimentar) quando não se consegue alimentar de maneira correta o rebanho. Senna \& Restle (1993) mostraram que fatores genéticos influenciam a $\mathrm{CC}$ dos animais nos diferentes períodos do ano, o que não foi verificado neste trabalho, provavelmente pelo fato dos animais não receberem alimentação necessária para expressarem suas características genéticas.

As correlações entre as características estudadas estão na Tabela 3. Em geral, as correlações das características estudadas são baixas e, às vezes, negativas. Os parâmetros RPN e RPD têm correlações médias e positivas com PBN e PBD respectivamente. Isto já era esperado, uma vez que as fórmulas para calcular estas características incluem estes pesos. As correlações destes parâmetros com o peso da vaca e o IEP são negativas e baixas. O RPN e o RPD só avaliam os pesos dos animais ao nascer ou ao desmame, ignorando informações de outras características de importância econômica.

As correlações da FR com o peso do bezerro são médias e positivas, enquanto com os pesos da vaca são médias/altas e positivas e têm correlação negativa e alta com o IEP, o que era esperado.

As correlações entre pesos do bezerro e pesos e CCs da vaca foram médias a altas $(0,19$ a 0,65$)$, indicando que vacas maiores produziram bezerros maiores ao nascer e no desmame.

Tabela 3 - Correlações entre as características reprodutivas de gado mestiço de corte no sistema da EMBRAPA Cerrados Table 3 - Correlations between reproductive traits in a mixed beef herd in the EMBRAPA Cerrados system

\begin{tabular}{|c|c|c|c|c|c|c|c|c|c|c|}
\hline & $\begin{array}{l}\text { PVD } \\
W D C\end{array}$ & $\begin{array}{l}\text { CCVD } \\
B C D W\end{array}$ & $\begin{array}{l}\text { PBD } \\
W C W\end{array}$ & $\begin{array}{r}\text { IEP } \\
C I\end{array}$ & $\begin{array}{l}\text { FR } \\
T F\end{array}$ & $\begin{array}{l}\mathrm{RPN} \\
R B W\end{array}$ & $\begin{array}{l}\text { RPD } \\
R W W\end{array}$ & $\begin{array}{l}\text { PVP } \\
W D C\end{array}$ & $\begin{array}{l}\text { CCVP } \\
B C D C\end{array}$ & $\mathrm{RPD}^{2}$ \\
\hline $\begin{array}{l}\mathrm{PBN} \\
B W C\end{array}$ & 0,48 & 0,19 & 0,36 & $-0,14$ & 0,27 & 0,46 & 0,07 & 0,44 & 0,24 & 0,11 \\
\hline $\begin{array}{l}\text { PVD } \\
W D C\end{array}$ & & 0,65 & 0,40 & $-0,25$ & 0,65 & $-0,12$ & $-0,05$ & 0,58 & 0,21 & $-0,21$ \\
\hline $\begin{array}{l}\text { CCVD } \\
B C D W\end{array}$ & & & 0,21 & $-0,21$ & 0,46 & $-0,03$ & $-0,03$ & 0,25 & 0,19 & $-0,21$ \\
\hline $\begin{array}{l}\text { PBD } \\
W C W\end{array}$ & & & & $-0,05$ & 0,19 & $-0,11$ & 0,64 & 0,26 & 0,11 & 0,82 \\
\hline $\begin{array}{l}\text { IEP } \\
C I\end{array}$ & & & & & $-0,82$ & $-0,06$ & $-0,01$ & $-0,09$ & 0,07 & 0,13 \\
\hline $\begin{array}{l}\text { FR } \\
T F\end{array}$ & & & & & & $-0,06$ & 0,00 & 0,41 & 0,05 & $-0,20$ \\
\hline $\begin{array}{l}\text { RPN } \\
R B W\end{array}$ & & & & & & & 0,35 & $-0,30$ & $-0,19$ & 0,20 \\
\hline $\begin{array}{l}\text { RPD } \\
R W W\end{array}$ & & & & & & & & $-0,26$ & $-0,18$ & 0,79 \\
\hline $\begin{array}{l}\text { PVP } \\
W D C\end{array}$ & & & & & & & & & 0,60 & $-0,10$ \\
\hline $\begin{array}{l}\text { CCVP } \\
B C D P\end{array}$ & & & & & & & & & & $-0,01$ \\
\hline
\end{tabular}

PVD = peso da vaca ao desmame; CCVD = condição corporal da vaca ao desmame; $P B D=$ peso do bezerro ao desmame; IEP= intervalo de partos; $\mathrm{FR}$ = fertilidade real; RPN = razão peso ao nascer do bezerro e peso da vaca no parto; RPD = razão peso do bezerro ao desmame e peso da vaca no parto; PVP = peso da vaca ao parto; CCVP = condição corporal da vaca no parto, $\mathrm{PBN}=$ peso do bezerro no nascer; RPD ${ }^{2}=$ razão peso do bezerro ao desmame e peso da vaca no desmame.

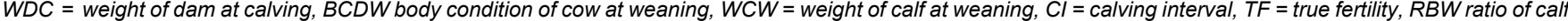
birth weight to cow weight at calving, RWW = ratio of calf weight at weaning to cow weight at calving, WDC= weight of dam at calving, $B C D C=$ body condition of dam at calving, BWC = birth weight of calf, $R W W^{2}=$ ratio of calf weight at weaning to cow weight at weaning. 
Os primeiros dois autovetores e os primeiros seis da análise dos componentes principais são mostrados na Figura 1 e Tabela 4 respectivamente. O primeiro autovetor mostra a importância das características do bezerro no conjunto de variáveis examinadas, enquanto o segundo mostra a importância das características da vaca. O primeiro autovetor contrasta os pesos da vaca e do bezerro no desmame e no nascimento com o IEP, mostrando que quando existe um aumento destes pesos, diminui o intervalo. $\mathrm{O}$ peso negativo do IEP neste componente é pequeno, também enfatizando o peso do bezerro no desmame dentro da fórmula para fertilidade real(FR). A CC da vaca no parto e desmame está relacionada positivamente com os pesos da vaca e do bezerro ao nascer e no desmame e com as razões entre o peso da vaca e do bezerro. O segundo autovetor contrasta a RPN e a RPD e as características da vaca ao parir. A mais alta, o peso e CC da vaca, a mais baixa, o RPD e RPN, o que era para ser esperado, mas o peso da vaca é o fator com mais ênfase dentro da conjuntura. A contribuição do peso do bezerro no desmame foi praticamente nula neste autovector. Os dois primeiros componentes respondem por $56 \%$ da variação total entre as 11 características examinadas, e os primeiros cinco explicam mais de $91 \%$ da variação total.

Os outros componentes (Tabela 4) mostraram relações diferentes entre as características originais, explicando uma quantidade cada vez menor da variação.

Tabela 4 - Os primeiros seis componentes principais das características reprodutivas do rebanho mestiço de corte da EMBRAPA Cerrados

Table 4 - The first six principal components of reproductive traits in mixed beef herd of EMBRAPA Cerrados

\begin{tabular}{|c|c|c|c|c|c|c|}
\hline & Prin1 & Prin2 & Prin 3 & Prin4 & Prin5 & Prin6 \\
\hline RPD* & 0,4246 & $-0,3249$ & $-0,1387$ & 0,0371 & 0,1858 & 0,0971 \\
\hline RPN & 0,2483 & $-0,1294$ & 0,5319 & $-0,4456$ & $-0,0680$ & 0,1371 \\
\hline RPD2 & 0,3867 & $-0,3469$ & $-0,0334$ & 0,2890 & $-0,0560$ & 0,0680 \\
\hline FR & 0,4374 & 0,1242 & $-0,3200$ & $-0,0984$ & $-0,2283$ & $-0,0777$ \\
\hline PVD & 0,1912 & 0,4941 & $-0,0604$ & $-0,0752$ & 0,3484 & $-0,2794$ \\
\hline PVP & 0,1141 & 0,4780 & 0,1316 & 0,3727 & $-0,0931$ & $-0,3386$ \\
\hline CCVD & 0,1175 & 0,3563 & $-0,1695$ & $-0,2352$ & 0,4875 & $0, .6081$ \\
\hline IEP & $-0,1220$ & $-0,1867$ & 0,4090 & 0,4120 & 0,6110 & $-0,0470$ \\
\hline CCVP & 0,0299 & 0,2717 & 0,1816 & 0,4906 & $-0,3610$ & 0,6171 \\
\hline PBN & 0,3045 & 0,1761 & 0,5821 & $-0,1938$ & $-0,1222$ & $-0,0872$ \\
\hline PBD & 0,4953 & $-0,0516$ & $-0,0739$ & 0,2429 & 0,1390 & $-0,0899$ \\
\hline \% variação & 0,3213 & 0,2415 & 0,1368 & 0,1330 & 0,0799 & 0,0630 \\
\hline
\end{tabular}

* Para siglas ver Tabela 2 ( ${ }^{\star}$ for symbols see Table 2 ).

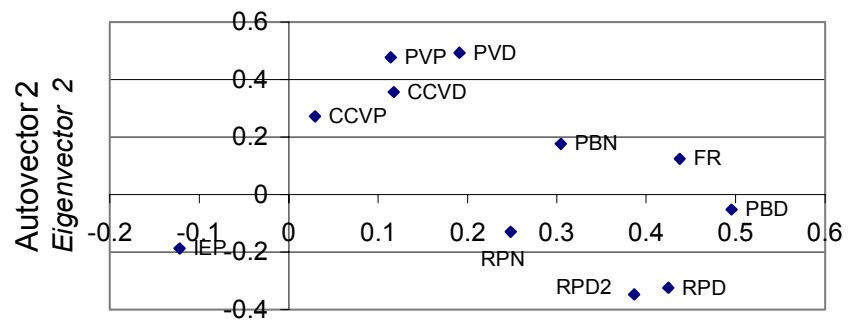

Autovector 2

Eigenvector 2

Figura 1 - Componentes principais das características reprodutivas do rebanho mestiço de corte da EMBRAPA Cerrados.

Figure 1 - Principal components of reproductive traits of the mixed beef herd of EMBRAPA Cerrados.

\section{Conclusões}

O índice de FR parece ser o melhor indicador de produtividade do rebanho tendo correlações mais altas com o IEP, peso da vaca e do bezerro enquanto a RPN e a RPD só possuem correlações médias com o peso do bezerro e não com as outras características reprodutivas do rebanho. O rebanho apresentou bons índices reprodutivos, principalmente quando se analisou o parâmetro mais utilizado atualmente para avaliação, o IEP. Porém, quando se utilizou maior conjunto de parâmetros, como a FR, a RPN e a RPD, observaram-se falhas que antes não eram vislumbradas. 
Os valores encontrados para esses parâmetros mostram um rebanho produtivo, porém com falhas a serem corrigidas, principalmente no que diz respeito ao peso de desmame e à condição corporal, que estão abaixo do ideal para que o rebanho expresse todo seu potencial produtivo e reprodutivo. A condição corporal foi importante na influência sobre fatores produtivos no rebanho estudado, mostrando a necessidade do controle da condição corporal para a melhoria dos índices produtivos e reprodutivos. Nas condições deste estudo, pode-se verificar que a mortalidade de bezerros pode ser baixa em rebanhos criados extensivamente. Verificou-se que a alimentação é um fator fundamental no desenvolvimento dos bezerros e o uso de touros ou sêmen europeus, nestas condições, não necessariamente leva à maior produtividade do rebanho.

\section{Literatura Citada}

ALENCAR, M.M.; BEOLCHI, E.A.; COSTA, J.L. et al. Intervalo entre partos de vacas Canchim. Pesquisa Agropecuária Brasileira, v.19, n.2, p.237-241, 1984.

ALENCAR, M.M. Desempenho produtivo de fêmeas das raças Canchim e Nelore. V. Desenvolvimento dos bezerros. Revista da Sociedade Brasileira de Zootecnia, v. 17, n.5, p.411-420, 1988.

ALENCAR, M.M.; TREMATORE, R.L.; OLIVEIRA, J.A.L. et al. Desempenho produtivo de vacas da raça nelore e cruzadas Charolês x Nelore, Limousin x Nelore e Tabapuã x Gir. Revista Brasileira de Zootecnia, v.26, n.3, p.467-472, 1997.

AYALA, J.M.N.; OLIVEIRA, H.N.; PEREIRA, J.C.C. Efeitos genéticos e não genéticos sobre características ponderais de duas populações de raça Nelore. Arquivo Brasileiro de Medicina Veterinária e Zootecnia, v.43, n.1, p.81-92, 1991.

BENEVIDES, L.M.S.; LÔBO, R.B.; BENEVIDES FILHO, I.M.F. Influência do mês e ano do parto e grupamento genético sobre a idade ao primeiro parto, intervalo de partos e número de crias de vacas Red Poll x Zebu no Estado de São Paulo. Revista Brasileira de Ciências Veterinária, v.4, n.1, p.25-29, 1997.

CAMPELLO, C.C.; MARTINS FILHO, R.; LOBO, R.N.B. Intervalo de partos e fertilidade real em vacas Nelore no Estado do Maranhão. Revista Brasileira de Zootecnia, v.28, n.3, p.474-479, 1999.

CARVALHO, F.A.N. A saga do Simental no Brasil. Curitiba: Mediograf, 1998. p.4.

EUCLIDES FILHO, K.; FIGUEIREDO, G.R.; THIAGO, L.R.L.S. Eficiência biológica de produção de carne de diferentes grupos genéticos. In: REUNIÃO ANUAL DA SOCIEDADE BRASILEIRA DE ZOOTECNIA, 29., 1992, Lavras. Anais... Lavras: Sociedade Brasileira de Zootecnia, 1992. p.124.
FRIES, L.A.; ALBUQUERQUE, L. Prenhez aos quatorze meses: presente e futuro. 1999. http://www.sbz.org.br/ eventos/PortoAlegre/homepagesbz/Fries.htm

GONÇALVES, F.H.D.; TORRES, C.A.A.; VETROMILA, M.A.M. Avaliação da fertilidade real e da capacidade mais provável de fertilidade real de matrizes de um rebanho da raça nelore. In: REUNIÃO ANUAL DA SOCIEDADE BRASILEIRA DE ZOOTECNIA, 33., 1996, Fortaleza. Anais... Fortaleza: Sociedade Brasileira de Zootecnia, 1996, p.368.

GONZALEZ, F.H.D.; TORRE, C.A.A.; VETROMILA, M.A.M. Efeito da condição corporal em novilhas mestiças sobre a fertilidade e os níveis de glicose, albumina e progesterona pós-serviço. In: REUNIÃO DA SOCIEDADE BRASILEIRA DE ZOOTECNIA, 30., 1993, Rio de Janeiro. Anais... Rio de Janeiro: Sociedade Brasileira de Zootecnia, 1993, p.439-444.

MARTINS, C.A.; SALOMONI, F. Efeito do sexo e do genótipo da progênie no desempenho reprodutivo subseqüente de vacas Hereford. Revista Brasileira de Reprodução Animal, v.14, n.3, p.187-199, 1990.

PEREIRA, J.C.C.; AYALA, J.M.N.; OLIVEIRA, H.N. Efeitos genéticos e não genéticos sobre idade ao primeiro parto e o intervalo entre partos de duas populações de raça nelore. Arquivo Brasileiro de Medicina Veterinária e Zootecnia, v.43, n.1, p.93-102, 1991.

RANDEL, R.D. Nutrition and postpartum rebreeding in cattle. Journal Animal Science, v.68, p.853-862, 1990.

Statistical Analysis System - SAS. User's Guide Version 8. Cary: 1999. 295p.

SEMMELMANN, C.E.N.; LOBATO, J.F.P.; ROCHA, M.G. Efeito de sistemas de alimentação no ganho de peso e desempenho reprodutivo de novilhas Nelore acasaladas aos 17/18 meses de idade. Revista Brasileira de Zootecnia, v.30, n.3, p.835-843, 2001.

SENNA, D.B.; RESTLE, J. Variação de peso de vacas de corte de quatro grupos genéticos submetidos a diferentes seqüências de pastagem cultivada. In: REUNIÃO DA SOCIEDADE BRASILEIRA DE ZOOTECNIA, 30., 1993, Rio de Janeiro. Anais... Rio de Janeiro: Sociedade Brasileira de Zootecnia, 1993, p.229.

SZÉCHY, M.L.M., BENEVIDES FILHO, I.M., SOUZA, L.M. Idade ao primeiro parto, intervalo de partos e peso ao nascimento de um rebanho Nelore. Revista Brasileira de Ciências Veterinária, v.2, n.2, p.47-49, 1995.
Recebido em: 16/06/00 Aceito em: 12/11/01 